\title{
Transcriptional factor regulation network and competitive endogenous RNA (ceRNA) network determining response of esophageal squamous cell carcinomas to neoadjuvant chemoradiotherapy
}

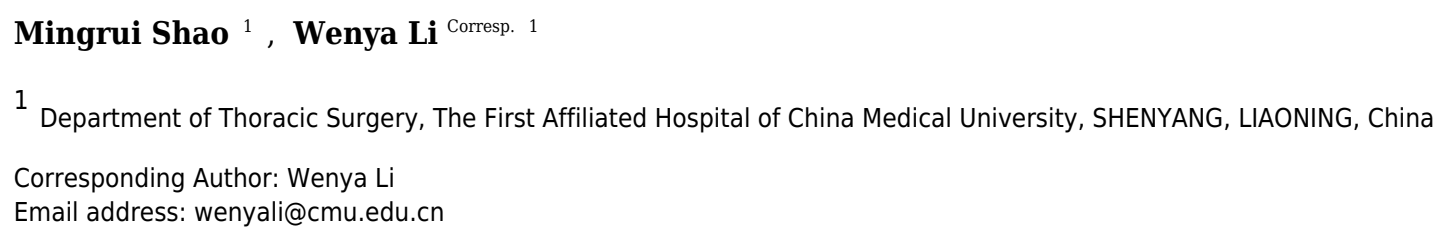

Background: Neoadjuvant chemoradiotherapy (nCRT) followed by surgery benefits survival for patients with esophageal squamous cell carcinomas (ESCC) compared with surgery alone, but the clinical outcomes of nCRT are heterogeneous. This study aimed to elucidate transcriptional factor (TF) regulation network and competitive endogenous RNA (ceRNA) network determining response of ESCC to $\mathrm{nCRT}$. Materials and Methods: RNA microarray data of GSE59974 and GSE45670 were analyzed to investigate the significant changes of lincRNAs, miRNAs, mRNAs in responders and non-responders of nCRT in ESCC. Functional and enrichment analyses were conducted by clusterProfiler. The target lincRNAs and mRNAs of miRNAs were predicted by miRWalk. The ceRNA and TF regulatory networks were constructed using Cytoscape. Results: Differentially expressed genes between responders and non-responders mainly enriched in biological process including Wnt signaling pathway and regulation of cell development and morphogenesis involved in differentiation. Besides, these genes showed enrichment in molecular function of glycosaminoglycan binding, metalloendopeptidase inhibitor and growth factor activity. KEGG analysis enriched these genes in pathways of neurotrophin signaling pathway, cell adhesion molecules and Wnt signaling pathway. We also constructed ceRNA network and TF network regulating response of ESCC to nCRT. Core regulatory miRNAs were miR-520a, miR-548am, miR-3184, miR-548d, miR-4725, miR-148a, miR-4659a and key regulatory TFs included MBNL1, SLC26A3, BMP4, ZIC1 and ANKRD7. Conclusion: We identified significantly altered lincRNAs, miRNAs and mRNAs involved in the nCRT response of ESCC. In addition, the ceRNA regulatory network of lincRNA-miRNA-mRNA and TF regulatory network were constructed, which would elucidate novel molecular mechanisms determining nCRT response of ESCC, thus providing promising clues for clinical therapy. 
1 Transcriptional factor regulation network and competitive endogenous RNA

2 (ceRNA) network determining response of esophageal squamous cell carcinomas

3 to neoadjuvant chemoradiotherapy

4 Author:

$5 \quad$ Mingrui Shao ${ }^{1}$, Wenya Li ${ }^{1}$

6 1Department of Thoracic Surgery, The First Affiliated Hospital of China Medical

7 University, Shenyang, Liaoning Province, China. Zip Code: 110001.

9 Corresponding author

10 Prof Wen-ya Li, Department of Thoracic Surgery, The First Affiliated Hospital of China

11 Medical University, NO.155 North Nanjing Street, Heping District, Shenyang, Liaoning

12 Province, China. Zip Code: 110001.

13 E-mail: saint5288@hotmail.com, Phone: 86-13514288257, 


\section{Abstract}

Background: Neoadjuvant chemoradiotherapy (nCRT) followed by surgery benefits survival for patients with esophageal squamous cell carcinomas (ESCC) compared with surgery alone, but the clinical outcomes of nCRT are heterogeneous. This study aimed to elucidate transcriptional factor (TF) regulation network and competitive endogenous RNA (ceRNA) network determining response of ESCC to nCRT.

Materials and Methods: RNA microarray data of GSE59974 and GSE45670 were analyzed to investigate the significant changes of lincRNAs, miRNAs, mRNAs in responders and non-responders of nCRT in ESCC. Functional and enrichment analyses were conducted by clusterProfiler. The target lincRNAs and mRNAs of miRNAs were predicted by miRWalk. The ceRNA and TF regulatory networks were constructed using Cytoscape.

Results: Differentially expressed genes between responders and non-responders mainly enriched in biological process including Wnt signaling pathway and regulation of cell development and morphogenesis involved in differentiation. Besides, these genes showed enrichment in molecular function of glycosaminoglycan binding, metalloendopeptidase inhibitor and growth factor activity. KEGG analysis enriched these genes in pathways of neurotrophin signaling pathway, cell adhesion molecules and Wnt signaling pathway. We also constructed ceRNA network and TF network regulating response of ESCC to $\mathrm{nCRT}$. Core regulatory miRNAs were miR-520a, miR-548am, miR3184, miR-548d, miR-4725, miR-148a, miR-4659a and key regulatory TFs included 
MBNL1, SLC26A3, BMP4, ZIC1 and ANKRD7.

37 Conclusion: We identified significantly altered lincRNAs, miRNAs and mRNAs involved

38 in the nCRT response of ESCC. In addition, the ceRNA regulatory network of lincRNA-

39 miRNA-mRNA and TF regulatory network were constructed, which would elucidate novel

40 molecular mechanisms determining nCRT response of ESCC, thus providing promising

41 clues for clinical therapy.

Key words: esophageal squamous cell carcinoma, transcriptional factor, ceRNA, neoadjuvant chemoradiotherapy. 
47

48

49

50

51

\section{Introduction}

As a common malignant tumour of upper digestive tract, esophageal squamous cell carcinoma (ESCC) represents a significant health burden worldwide due to its aggressive growth(Kang et al. 2015). There is accumulating evidence that surgical resection of locally advanced ESCC is an effective method to control the progression of this disease(Sjoquist et al. 2011). In some cases, however, the recurrence after curative resection and unsatisfactory survival status still pose significant obstacle for surgeons(Rohatgi et al. 2006). In recent years, it has been proved that neoadjuvant chemoradiotherapy (nCRT) followed by surgery benefits survival for patients with ESCC compared with surgery alone, which is recommended in the guidelines for ESCC therapy(Allum et al. 2011). Currently, up to one-third of patients show a pathologically complete response after neoadjuvant chemoradiotherapy. To optimise the efficacy of neoadjuvant treatment for individual patients, prediction of response to neoadjuvant treatment is highly desired(Eyck et al. 2018).

Noncoding RNAs (ncRNAs) are transcripts which possess no protein coding potential, the number of which constitutes over $98 \%$ of the entire genome transcripts(Anastasiadou et al. 2018)(Anastasiadou et al. 2018)(Anastasiadou et al. 2018). lincRNAs utilize a variety of mechanisms to translationally modulate protein expression, degradation and modification, of which the most critical regulation is competing endogenous RNAs (ceRNAs) theory proposed by Salmena $L$ et al. in 2011(Salmena et al. 2011). The hypothesis of ceRNA described complex 
posttranscriptional communication network of all transcript RNA species including lincRNAs, which can act as natural miRNA sponges to inhibit miRNA functions by sharing miRNA response elements (MRE)(Giroud \& Scheideler 2017). Emerging evidence has confirmed the importance of the ceRNA regulatory network of lincRNA-miRNA-mRNA in the initiation and progression of ESCC(Li et al. 2017; Shang et al. 2018; Yang et al. 2016). Transcriptional factor (TF) is a group of protein molecules that can specifically bind to the certain sequence of the $5^{\prime}$ end of the gene, thereby ensuring that the target gene is expressed in a specific time and space with a specific intensity(Battaglia et al. 2010). Normal transcription displays a high degree of flexibility over the choice, timing and magnitude of mRNA expression levels, which determines various biological processes including cancer(Zhong \& Ye 2014). A number of transcriptional factors have been suggested to participate in the regulation of ESCC(Pan et al. 2017; Zhu et al. 2014; Zhu et al. 2016). But no specific TF regulatory network has been drawn to outline the whole complex processes of nCRT response determination in ESCC.

Although nCRT followed by surgery has shown great advantages, the clinical outcomes of ESCC nCRT are heterogeneous(Rohatgi et al. 2005). Favourable survival is consistently achieved in patients who obtain pathologic complete response instead of the nonresponders(Donahue et al. 2009). Thus, understanding the underlying determinants of nCRT response could largely improve ESCC management. Despite considerable improvement in the understanding of lincRNAs and miRNAs, only a fraction of annotated ones has been well identified for biological function in ESCC. Until now, the whole picture 
89 of the ceRNA regulatory mechanism of lincRNA-miRNA-mRNA in the determination of

90 response of ESCC to $\mathrm{nCRT}$ still remains unclear. In addition, the complete TF regulation

91 network of nCRT response in ESCC is also elusive. In this study, we used RNA

92 microarray data to screen significantly altered lincRNAs, miRNAs and mRNAs in different

93 nCRT responses of ESCC. Besides, the ceRNA modulation and transcriptional factor

94 regulation networks were constructed, which would elucidate the molecular mechanisms

95 involved in the determination of $\mathrm{nCRT}$ response of ESCC, thus providing novel clues for

96 ESCC treatment.

97 Materials and Methods

98

99

100

101

102

103

104

\section{Microarray data}

Microarray data were obtained from Gene Expression Omnibus (GEO, https://www.ncbi.nlm.nih.gov/geo/) in NCBI (The National Center for Biotechnology Information). GSE59974 is a miRNA expression array of esophageal squamous cell carcinomas with different neoadjuvant chemoradiotherapy response. GSE45670 is an mRNA profiling dataset of esophageal squamous cell carcinomas with different neoadjuvant chemoradiotherapy response. Both GSE59974 and GSE45670 contained 17 responder samples and 11 nonresponder samples. Patients who received neo-CRT and surgery and had fresh pretreatment tissue specimens available for microarray were included. Patients with stage IIB-III squamous cell carcinoma according to the 6th AJCC Cancer Staging, which were technically resectable and medical operable, were considered to receive neo-CRT and surgery. Moreover, patients concurrently received 
110 vinorelbine (25 mg/m2) via intravenous injection on days $1,8,22$, and 29 , and cisplatin

111 (75 mg/ m2) via IV on days 1 and 22, or cisplatin (25 mg/m2) via IV daily on days 1 to 4

112 and 22 to 25 . Approximately 4 to 6 weeks after the end of CRT, patients underwent

113 attempted surgical resection of the primary tumor and regional nodes.

114 Data processing

115 GEO2R (http://www.ncbi.nlm.nih.gov/geo/geo2r/) is an interactive tool which can be used to compare two or more groups of samples to identify differentially expressed items

117 in GEO series. We adopted GEO2R to filter differentially expressed genes (DEGs) between responder and non-responder samples separately in each of the data sets. $P$ $<0.05$ and $|\log \mathrm{FC}|>1.5$ were considered as statistically significant. Duplicate gene probes and unspecific probes will be removed. In order to ensure the accuracy of the assay, we selected miRNAs with multiple probes and consistent expression trends as differential miRNAs.

\section{Gene ontology and pathway enrichment analyses} gene products. It contains terms under three categories: cellular component, molecular

126 function, and biological process(2006). Kyoto Encyclopedia of Genes and Genomes

127 (KEGG) is a collection of databases that contain information about genomes, biological pathways, diseases, and chemical substances(Kanehisa \& Goto 2000). In the present study, GO and KEGG pathway enrichment analyses were performed by using R package clusterprofiler. $\mathrm{P}<0.05$ was considered as statistically significant. 
131 Transcription factor regulation network

132 Search Tool for the Retrieval of Interacting Genes (STRING) database was used to

133 find interacting proteins between different genes(von Mering et al. 2005). Interactions with

134 a combined score > 0.4 were defined as significant. Next, we use CentiScape, an

135 application in Cytoscape, to screen for the hub protein. TFCheckpoint database was used

136 to identify the transcription factor among differentially expressed genes. The transcription

137 factor with connection numbers over 5 were considered as the hub transcription factors.

138 Using Cytoscape, we finally built transcription factor regulation network.

139

140

141

142

\section{MiRNA regulatory network}

The target genes of differentially expressed miRNAs were predicted by miRWalk (http://zmf.umm.uni-heidelberg.de/apps/zmf/mirwalk/index.html), which is a comprehensive online algorithm that provides information on miRNA from Human, Mouse and Rat on their predicted as well as validated binding sites on their target genes(Dweep et al. 2011). Accurate classification of $3 p / 5 p$ for miRNAs was adopted in target gene prediction. The predicted target genes were matched to the genes whose mRNA expressions were opposite to the miRNA profile because miRNAs are negatively regulated genes. In addition, the miRNAs with connection numbers more than 5 were selected as the hub miRNAs.

\section{CeRNA regulation network}

Using miRWalk, we predicted the interacrtion of lincRNA with miRNA. Then, we matched the targeted lincRNAs with the differentially expressed mRNAs in the mRNA 
152 expression array. In this study, the top 10 miRNA was used the built the ceRNA regulation

153 network. According to the ceRNA theory that lincRNAs act as natural miRNA sponges to

154 inhibit miRNA functions, the expressions of lincRNA-miRNA and miRNA-mRNA were all

155 negatively correlated. Finally, the ceRNA regulatory network of lincRNA-miRNA-mRNA

156 in nCRT response of ESCC was constructed by Cytoscape software. The significantly

157 altered miRNAs/mRNAs were analyzed for predictive power in separating responders

158 from non-responders.

159 Results

Identification of DEGs in esophageal squamous cell carcinomas with different neoadjuvant chemoradiotherapy response

The flow chart to visualize the analyses and define/explain the input and output was

summarized in Supplementary Figure 1. After differential expression analysis, a total of 61 genes in GSE45670 showed decreased expression in responder group and 84 genes were up-regulated in responder group (Supplementary Figure 2). As for GSE59974 dataset, a total of 247 differentially expressed miRNAs were identified, 110 of which were down-regulated and 137 were up-regulated in responders (Supplementary Figure 3). The heatmap of the two arrays were shown in Figure 1. Heatmap of the hub genes in the regulation network were shown in Supplementary Figure 4. All differential expression genes were showed in the supplemental table 1

171 GO and pathway functional enrichment analyses We then performed GO functional enrichment analysis of differentially expressed 
173 genes between responders and non-responders. The results showed that the

174 differentially expressed gene mainly enriched in biological process (BP) including positive

175 regulation of cell morphogenesis involved in differentiation, negative regulation of Wnt 176 signaling pathway and positive regulation of cell development. Cellular component (CC)

177 analysis indicated enrichment in proteinaceous extracellular matrix, cornified envelope

178 and basolateral plasma membrane. Besides, these DEGs showed significant enrichment

179 in molecular function (MF) of glycosaminoglycan binding, metalloendopeptidase inhibitor

180 activity and growth factor activity (Table 1, Figure 2). KEGG analysis enriched these

181 DEGs in pathways of Neurotrophin signaling pathway, Cell adhesion molecules and Wnt 182 signaling pathway (Table 2, Figure 3).

183 Transcription factor regulation network

According to STRING dataset, totally 49 proteins interacted with each other. Using CentiScape software, we selected BMP4 as the hub transcription factors (Table 3). We then built the transcription factor regulation network. As was shown in Figure 4, the red node represents the up-regulated gene and the blue node represents the down-regulated gene. The darker the color, the higher the expression level was. Purple border represented transcription factors. The node size increased with degree.

MiRNA regulation network

In the miRNA regulation network, a total of 13 genes were regulated by up-regulated hub miRNAs (Figure 5A), and 31 genes were regulated by the hub miRNAs with

193 decreased expression (Figure 5B). Subsequently, we selected the miRNAs with 
194 connection numbers $>5$ as the hub miRNA, which were hsa-miR-520a-3p, hsa-miR-

195 548am-3p, hsa-miR-3184-5p, hsa-miR-548d-5p, hsa-miR-4725-3p, hsa-miR-148a-5p,

196 and hsa-miR-4659a-3p (Table 3).

\section{CeRNA regulation network}

198 Totally 15 non-coding RNAs showed significant differences in the expression profiling, 7 of which were up-regulated while the others were down-regulated. After matching the predicted results, altogether 12 lincRNAs were associated with the differentially expressed miRNAs (Table 4). APCDD1L-AS1 had the highest degree in the overexpression group while degree of SOX2-OT was the highest in the decreased expression group. The ceRNA regulatory network of lincRNA-miRNA-mRNA in nCRT

response of ESCC was shown in Figure 6. In addition, the AUC (area under curve),

sensitivity and specificity of significantly altered miRNA/mRNAs were summarized in

206 Table 5.

207

208

209

210

211

212

\section{Discussion}

Although nCRT before surgery has been accepted as standard strategy for locally advanced ESCC, it is still difficult for surgeons to predict whether the patient present favourable response to $\mathrm{nCRT}$. In order to understand the underlying determinants of nCRT response of ESCC patients, miRNA expression array (GSE59974) and mRNA profiling dataset (GSE45670) of ESCC with different nCRT responses were systematically analyzed. The pretreatment samples were adopted for microarray, the results of which indicate predictive network for nCRT response. Finally, the ceRNA 
215 modulation and transcriptional factor regulation networks were constructed, which would

216 contribute to the understanding of molecular mechanisms involved in the determination

217 of $\mathrm{nCRT}$ response of ESCC.

218 A total of 61 genes showed decreased expression in responder group and 84 genes

219 were up-regulated in responder group in the present study. According to the functional

220 enrichment analysis, DEGs between responders and non-responders were mainly

221 enriched in biological process including regulation of cell morphogenesis involved in

222 differentiation, Wnt signaling pathway, cell development and protein import. Additionally,

223 these DEGs showed significant enrichment in molecular function of glycosaminoglycan

224 binding, metalloendopeptidase inhibitor activity, receptor serine, growth factor activity and

225 carboxylic acid binding. KEGG analysis enriched these DEGs in pathways of axon

226 guidance, metabolism of xenobiotics by cytochrome P450, neurotrophin signaling

227 pathway, cell adhesion molecules and Wnt signaling pathway. Wnt pathway has

228 previously been reported to play critical role in cell fate specification, cell proliferation and

229 cell migration during embryonic development and carcinogenesis(Duchartre et al. 2016).

230 Growth factor is a naturally occurring substance capable of stimulating cellular growth,

231 proliferation, healing, and cellular differentiation(Lappano et al. 2013). The results of this

232 study reveal promising novel functions of these substance or pathways in determining

233 nCRT response of ESCC.

234 Transcriptional factor (TF) is a group of protein molecules that can efficiently control

235 the expression of target gene in a specific time and space with a specific intensity(Swift 
236 \& Coruzzi 2017). With the help of STRING and CentiScape software, we built the

237 transcription factor regulation network which determines response of ESCC to

238 neoadjuvant chemoradiotherapy. As was shown in the TF regulation network, TFs such

239 as MBNL1, SLC26A3, BMP4, ZIC1 and ANKRD7 synergistically modulate the response

240 of ESCC patients to nCRT. It was worth noting that BMP4 was the hub transcription factor

241 in this regulation network with the highest degree. Previously, BMP4 was found to be

242 significantly up-regulated in ESCC than normal squamous epithelium(van Baal et al.

243 2008). In addition, BMP signaling pathways has been reported to affect differently

244 migration and invasion of esophageal squamous cancer cells(Hu et al. 2017). In this

245 study, our findings indicated that BMP4 might also play an important role in ESCC nCRT

246 response. Most of other TFs have never been linked to ESCC, which offer us potential

247 therapeutic targets to improve nCRT response of ESCC.

248 By means of miRWalk database, we constructed miRNA regulation network involved

249 in the nCRT response of ESCC including 13 genes regulated by up-regulated hub

250 miRNAs and 31 genes regulated by the hub miRNAs with decreased expression. The

251 miRNAs with connection numbers over 5 were defined as hub miRNAs, which were hsa-

252 miR-520a-3p, hsa-miR-548am-3p, hsa-miR-3184-5p, hsa-miR-548d-5p, hsa-miR-4725-

$2533 p$, hsa-miR-148a-5p, and hsa-miR-4659a-3p. Previously, miR-520a has been proved to

254 modulate the expression of ErbB4 and suppresses the proliferation and invasion of ESCC

255 cells in vitro, indicating its role as a tumor suppressor(Ye et al. 2014). Besides, miR-

256 148a was indicated to be implicated in carcinogenesis in primary ESCC through 
257 regulating HLA-G expression(Chen et al. 2017). Together with our findings in this study,

$258 \mathrm{miR}-520 \mathrm{a}$ and miR-148a might probably serve as key regulators of ESCC carcinogenesis

259 and nCRT response.

260 In order to construct the ceRNA regulatory network of lincRNA-miRNA-mRNA, we

261 next predicted the interaction of miRNAs with lincRNAs and mRNAs. According to the

262 ceRNAs theory, the expressions of miRNAs should be negatively correlated with

263 expressions of targeting lincRNAs and mRNAs. Therefore, we overlapped the predicted

264 targets of up-regulated Differentially Expressed miRNAs (DEMs) with down-regulated

265 Differentially Expressed lincRNAs (DELs) and DEGs, as well as overlapped the predicted

266 targets of down-regulated DEMs with up-regulated DELs and DEGs. Finally, the ceRNA

267 regulatory network of lincRNA-miRNA-mRNA in determining nCRT response of ESCC

268 was built, which contains 12 lincRNAs associated with the DEMs. APCDD1L-AS1 had the

269 highest degree in the overexpression group while degree of SOX2-OT was the highest in

270 the decreased expression group. These lincRNAs hold great potential as biomarkers of

271 predicting $\mathrm{nCRT}$ response of different ESCC patients.

272 During the past few years there has been a considerable progress in ESCC, where

273 it has been most extensively studied in nCRT therapy followed by surgery. However,

274 limited information was revealed about determination of nCRT response and few

275 validated biomarkers could guide ESCC chemotherapy or radiotherapy presently. The

276 identified significantly altered lincRNAs, miRNAs and mRNAs involved in the nCRT

277 response of ESCC in this study would offer physicians opportunities to save ineffective 
278 treatments and turn to alternative methods, thus avoiding the over- or under-treatment of

279 ESCC individuals. In addition, the ceRNA regulatory network of lincRNA-miRNA-mRNA

280 and transcriptional factor regulatory network we constructed would shed new light on the

281 molecular mechanisms determining nCRT response of ESCC. We should acknowledge

282 the limitation that no experiment was performed to confirm the findings, which should be

283 researched in the future.

\section{Conclusion}

We identified a series of significantly altered lincRNAs, miRNAs and mRNAs involved

in the $\mathrm{nCRT}$ response of ESCC by comparing RNA microarray data of responders and non-responders. In addition, the ceRNA regulatory network of lincRNA-miRNA-mRNA and transcriptional factor regulatory network were constructed, which would elucidate novel molecular mechanisms determining nCRT response of ESCC, thus providing promising clues for clinical therapy. Core regulatory miRNAs of miR-520a, miR-548am, miR-3184, miR-548d, miR-4725, miR-148a, miR-4659a and key regulatory transcriptional

factors of MBNL1, SLC26A3, BMP4, ZIC1 and ANKRD7 might provide biological insight into the full repertoire of nCRT response of ESCC.

Conflict of interest: All of the authors declare that there is no conflict of interest.

Funding: This study was supported by Natural Science Foundation of Liaoning Province

298 (grant no. 2015020561) and the Fund for Scientific Research of The First Hospital of 
299 China Medical University (grant no. fsfh1514).

300

301

302

303

304

305

306 
307

\section{Reference}

2006. The Gene Ontology (GO) project in 2006. Nucleic Acids Res 34:D322-326.

Allum WH, Blazeby JM, Griffin SM, Cunningham D, Jankowski JA, and Wong R. 2011. Guidelines for the management of oesophageal and gastric cancer. Gut 60:1449-1472.

Anastasiadou E, Jacob LS, and Slack FJ. 2018. Non-coding RNA networks in cancer. Nat Rev Cancer 18:5-18.

Battaglia S, Maguire O, and Campbell MJ. 2010. Transcription factor co-repressors in cancer biology: roles and targeting. Int J Cancer 126:2511-2519.

Chen Q, Luo G, and Zhang X. 2017. MiR-148a modulates HLA-G expression and influences tumor apoptosis in esophageal squamous cell carcinoma. Exp Ther Med 14:4448-4452.

Donahue JM, Nichols FC, Li Z, Schomas DA, Allen MS, Cassivi SD, Jatoi A, Miller RC, Wigle DA, Shen KR, and Deschamps C. 2009. Complete pathologic response after neoadjuvant chemoradiotherapy for esophageal cancer is associated with enhanced survival. Ann Thorac Surg 87:392-398; discussion 398-399.

Duchartre Y, Kim YM, and Kahn M. 2016. The Wnt signaling pathway in cancer. Crit Rev Oncol Hematol 99:141149.

Dweep H, Sticht C, Pandey P, and Gretz N. 2011. miRWalk--database: prediction of possible miRNA binding sites by "walking" the genes of three genomes. $J$ Biomed Inform 44:839-847.

Eyck BM, van der Wilk BJ, Lagarde SM, Wijnhoven BPL, Valkema R, Spaander MCW, Nuyttens J, van der Gaast A, and van Lanschot JJB. 2018. Neoadjuvant chemoradiotherapy for resectable oesophageal cancer. Best Pract Res Clin Gastroenterol 36-37:37-44.

Giroud M, and Scheideler M. 2017. Long Non-Coding RNAs in Metabolic Organs and Energy Homeostasis. Int $J$ Mol Sci 18.

Hu M, Cui F, Liu F, Wang J, Wei X, and Li Y. 2017. BMP signaling pathways affect differently migration and invasion of esophageal squamous cancer cells. Int J Oncol 50:193-202.

Kanehisa M, and Goto S. 2000. KEGG: kyoto encyclopedia of genes and genomes. Nucleic Acids Res 28:27-30.

Kang X, Chen K, Li Y, Li J, D'Amico TA, and Chen X. 2015. Personalized targeted therapy for esophageal squamous cell carcinoma. World J Gastroenterol 21:7648-7658.

Lappano R, De Marco P, De Francesco EM, Chimento A, Pezzi V, and Maggiolini M. 2013. Cross-talk between GPER and growth factor signaling. J Steroid Biochem Mol Biol 137:50-56.

Li Y, Chen D, Gao X, Li X, and Shi G. 2017. LncRNA NEAT1 Regulates Cell Viability and Invasion in Esophageal Squamous Cell Carcinoma through the miR-129/CTBP2 Axis. Dis Markers 2017:5314649.

Pan F, Mao H, Bu F, Tong X, Li J, Zhang S, Liu X, Wang L, Wu L, Chen R, Wei H, Li B, Li C, Yang Y, Steer CJ, Zhao J, and Guo Y. 2017. Sp1-mediated transcriptional activation of miR-205 promotes radioresistance in esophageal squamous cell carcinoma. Oncotarget 8:5735-5752.

Rohatgi P, Swisher SG, Correa AM, Wu TT, Liao Z, Komaki R, Walsh GL, Vaporciyan AA, Rice DC, Roth JA, and Ajani JA. 2005. Characterization of pathologic complete response after preoperative chemoradiotherapy in carcinoma of the esophagus and outcome after pathologic complete response. Cancer 104:2365-2372.

Rohatgi PR, Swisher SG, Correa AM, Wu TT, Liao Z, Komaki R, Walsh GL, Vaporciyan AA, Rice DC, Bresalier RS, Roth JA, and Ajani JA. 2006. Histologic subtypes as determinants of outcome in esophageal carcinoma patients with pathologic complete response after preoperative chemoradiotherapy. Cancer 106:552-558.

Salmena L, Poliseno L, Tay Y, Kats L, and Pandolfi PP. 2011. A ceRNA hypothesis: the Rosetta Stone of a hidden 
RNA language? Cell 146:353-358.

Shang M, Wang X, Zhang Y, Gao Z, Wang T, and Liu R. 2018. LincRNA-ROR promotes metastasis and invasion of esophageal squamous cell carcinoma by regulating miR-145/FSCN1. Onco Targets Ther 11:639-649.

Sjoquist KM, Burmeister BH, Smithers BM, Zalcberg JR, Simes RJ, Barbour A, and Gebski V. 2011. Survival after neoadjuvant chemotherapy or chemoradiotherapy for resectable oesophageal carcinoma: an updated metaanalysis. Lancet Oncol 12:681-692.

Swift J, and Coruzzi GM. 2017. A matter of time - How transient transcription factor interactions create dynamic gene regulatory networks. Biochim Biophys Acta 1860:75-83.

van Baal JW, Milana F, Rygiel AM, Sondermeijer CM, Spek CA, Bergman JJ, Peppelenbosch MP, and Krishnadath KK. 2008. A comparative analysis by SAGE of gene expression profiles of esophageal adenocarcinoma and esophageal squamous cell carcinoma. Cell Oncol 30:63-75.

von Mering C, Jensen LJ, Snel B, Hooper SD, Krupp M, Foglierini M, Jouffre N, Huynen MA, and Bork P. 2005. STRING: known and predicted protein-protein associations, integrated and transferred across organisms. Nucleic Acids Res 33:D433-437.

Yang S, Ning Q, Zhang G, Sun H, Wang Z, and Li Y. 2016. Construction of differential mRNA-lncRNA crosstalk networks based on ceRNA hypothesis uncover key roles of lncRNAs implicated in esophageal squamous cell carcinoma. Oncotarget 7:85728-85740.

Ye W, Yao Q, Zhang M, Wen Q, and Wang J. 2014. [miR-520a regulates ErbB4 expression and suppresses proliferation and invasion of esophageal squamous cell carcinoma]. Nan Fang Yi Ke Da Xue Xue Bao 34:164168.

Zhong R, and Ye ZH. 2014. Complexity of the transcriptional network controlling secondary wall biosynthesis. Plant Sci 229:193-207.

Zhu H, Chen X, Chen B, Chen B, Fan J, Song W, Xie Z, Jiang D, Li Q, Zhou M, Sun D, and Zhao Y. 2014. Activating transcription factor 4 mediates a multidrug resistance phenotype of esophageal squamous cell carcinoma cells through transactivation of STAT3 expression. Cancer Lett 354:142-152.

Zhu Y, Zhu MX, Zhang XD, Xu XE, Wu ZY, Liao LD, Li LY, Xie YM, Wu JY, Zou HY, Xie JJ, Li EM, and Xu LY. 2016. SMYD3 stimulates EZR and LOXL2 transcription to enhance proliferation, migration, and invasion in esophageal squamous cell carcinoma. Hum Pathol 52:153-163. 
377 Figure legends

378 Figure 1, Heatmap representing the expression levels of genes between responder and

379 non-responder samples. A, heatmap of the top 100 mRNA of GSE45670. B, heatmap of

380 the top 100 miRNA in GSE59974.

381 Figure 2, Gene ontology analysis of differentially expressed genes associated with

382 response of esophageal squamous cell carcinomas to neoadjuvant chemoradiotherapy.

383 A total of 145 mRNAs were chosen for GO analysis for biological process, cellular

384 component and molecular function. The size of circle represented the gene counts in go

385 terms, the color represented the $\mathrm{p}$ value of go terms. A, biological process. B, cellular

386 component. C, molecular function

387 Figure 3, KEGG pathway analysis of differentially expressed genes associated with

388 response of esophageal squamous cell carcinomas to neoadjuvant chemoradiotherapy.

389 A total of 145 mRNAs were chosen for kegg analysis. the color represented the $p$ value

390 of kegg terms.

391 Figure 4, Protein-protein interaction between differentially expressed genes. A total of 49

392 proteins interacted with each other. The size of the circle in the network represents the

393 number of connections, red represents an up-regulated gene, blue represents a down-

394 regulated expression gene, and purple frame represents a transcription factor.

395 Figure 5, MiRNA regulation network associated with response of esophageal squamous

396 cell carcinomas to neoadjuvant chemoradiotherapy. The size of the circle in the network

397 represents the number of connections. pink represents high expression, blue represents 
398 low expression. The fill color represents mRNA. Outer frame color represents miRNA. A,

39913 genes were regulated by two highly expressed hub miRNAs. B, 31 genes were 400 regulated by the 5 low expressed hub miRNAs.

401 Figure 6, A: Competitive endogenous RNA (ceRNA) regulation network associated with 402 response of esophageal squamous cell carcinomas to neoadjuvant chemoradiotherapy.

403 The size of the circle in the network represents the number of connections. pink 404 represents high expression, blue represents low expression. Diamonds represent 405 miRNAs. The fill color of the circle represents IncRNA. The outer frame color represents 406 mRNA. A, 6 highly expressed IncRNAs regulate 10 miRNAs and 44 genes. B, 6 highly 407 expressed IncRNAs regulate 10 miRNAs and 27 genes.

Supplementary Figure 1, The flow chart to visualize the analyses and explain the input 410 and output.

411 Supplementary Figure 2, Volcano plot of mRNA expression array, a total of 145 genes

412 have differences in expression. Green represents no difference, blue represents low 413 expression in the response group, and red represents high expression in the response 414 group.

415 Supplementary Figure 3, Volcano plot of miRNA expression array, a total of 247 miRNA 416 have differences in expression. Green represents no difference, blue represents low 417 expression in the response group, and red represents high expression in the response 418 group. 
419 Supplementary Figure 4, heatmap of the hub genes in the regulation network. A, 99 genes

420 in the regulation network in GSE45670. B, 21 miRNAs in regulation network in 421 GSE59974.

422

423

424

425

426

427 


\section{Table $\mathbf{1}$ (on next page)}

Gene ontology analysis of differentially expressed genes associated with response of esophageal squamous cell carcinomas to neoadjuvant chemoradiotherapy

Results of the top five Biological Process, Cellular Component and Molecular Function 


\begin{tabular}{|c|c|c|c|c|c|}
\hline Category & GO ID & Term & Count & $\%$ & $\mathbf{P}$ \\
\hline $\mathrm{BP}$ & GO:0044236 & multicellular organism metabolic process & 6 & 5.71 & 0.000198 \\
\hline $\mathrm{BP}$ & GO:0010770 & positive regulation of cell morphogenesis involved in differentiation & 5 & 4.76 & 0.001407 \\
\hline $\mathrm{BP}$ & GO:0030178 & negative regulation of Wnt signaling pathway & 4 & 3.81 & 0.034422 \\
\hline $\mathrm{BP}$ & GO:0010720 & positive regulation of cell development & 8 & 7.62 & 0.007031 \\
\hline $\mathrm{BP}$ & GO:0042307 & positive regulation of protein import & 3 & 2.86 & 0.02106 \\
\hline $\mathrm{CC}$ & GO:0005578 & proteinaceous extracellular matrix & 9 & 8.04 & 0.000389 \\
\hline $\mathrm{CC}$ & GO:0001533 & cornified envelope & 4 & 3.57 & 0.000622 \\
\hline $\mathrm{CC}$ & GO:0016323 & basolateral plasma membrane & 5 & 4.46 & 0.008398 \\
\hline $\mathrm{CC}$ & GO:0030424 & axon & 7 & 6.25 & 0.009971 \\
\hline $\mathrm{CC}$ & GO:0055037 & recycling endosome & 4 & 3.57 & 0.015016 \\
\hline MF & GO:0005539 & glycosaminoglycan binding & 7 & 6.80 & 0.000223 \\
\hline MF & GO:0008191 & metalloendopeptidase inhibitor activity & 2 & 1.94 & 0.003633 \\
\hline MF & GO:0033612 & receptor serine & 2 & 1.94 & 0.003633 \\
\hline MF & GO:0008083 & growth factor activity & 4 & 3.88 & 0.017013 \\
\hline MF & GO:0031406 & carboxylic acid binding & 4 & 3.88 & 0.019548 \\
\hline
\end{tabular}

Abbreviation: BP, Biological Process. CC, Cellular Component. MF, Molecular Function. 


\section{Table 2(on next page)}

KEGG analysis for differentially expressed genes associated with response of esophageal squamous cell carcinomas to neoadjuvant chemoradiotherapy.

Result of the pathway analysis of differentially expressed genes 
1

\begin{tabular}{cccccc}
\hline ID & Description & Count & $\mathbf{\%}$ & P & Genes \\
\hline hsa04360 & Axon guidance & 5 & 12.19512 & 0.002621 & EPHB2, L1CAM, CAMK2B, NRP1, PAK5 \\
hsa00980 & Metabolism of xenobiotics by cytochrome P450 & 3 & 7.317073 & 0.007782 & AKR1C1, EPHX1, ADH1B \\
hsa04722 & Neurotrophin signaling pathway & 3 & 7.317073 & 0.027708 & NGF, CAMK2B, RPS6KA6 \\
hsa04514 & Cell adhesion molecules (CAMs) & 3 & 7.317073 & 0.045651 & CLDN8, L1CAM, CDH2 \\
hsa04310 & Wnt signaling pathway & 3 & 7.317073 & 0.046434 & CAMK2B, WIF1, SOST \\
\hline
\end{tabular}

2 


\section{Table 3(on next page)}

Hub transcription factor and miRNA associated with response of esophageal squamous cell carcinomas to neoadjuvant chemoradiotherapy

One gene and seven miRNA were selected as hub 


\begin{tabular}{cccc}
\hline gene & ID & Targeting mRNAs & Change \\
\hline BMP4 & 652 & ZIC1, SOST, ALB, TF, CDH2, PTHLH & Down \\
hsa-miR-520a-3p & MIMAT0002834 & BAGE, ELAVL2, GPR158, INHBA, KLK5, PTHLH & Down \\
hsa-miR-548am-3p & MIMAT0019076 & CTAG2, DNAH5, GLCC1, QSOX2, RHEBL1, RSPO2, SEZ6L2 & Down \\
hsa-miR-3184-5p & MIMAT0015064 & C1QTNF6, CDSN, IQGAP2, RRBP1, SEZ6L2, TNNT1, WFDC12 & Down \\
hsa-miR-548d-5p & MIMAT0004812 & CDH18, COL12A1, DDAH1, KRT37, PLEKHS1, PPP4R4, PSG5, SKP2 & Down \\
hsa-miR-4725-3p & MIMAT0019844 & C1QTNF6, EPHA10, FCHSD1, PLEC, PLEKHS1, QSOX2, RHEBL1, & Down \\
hsa-miR-148a-5p & MIMAT0004549 & WBDC12 & Up \\
hsa-miR-4659a-3p & MIMAT0019727 & CHP2, CLDN8, CYP26A1, FAXC, JAKMIP3, KLF12, MBNL1, SCUBE2 & Up \\
\hline
\end{tabular}




\section{Table 4 (on next page)}

Differentially expressed IncRNA associated with response of esophageal squamous cell carcinomas to neoadjuvant chemoradiotherapy

Nine lincRNAs were selected as hub IncRNAs 


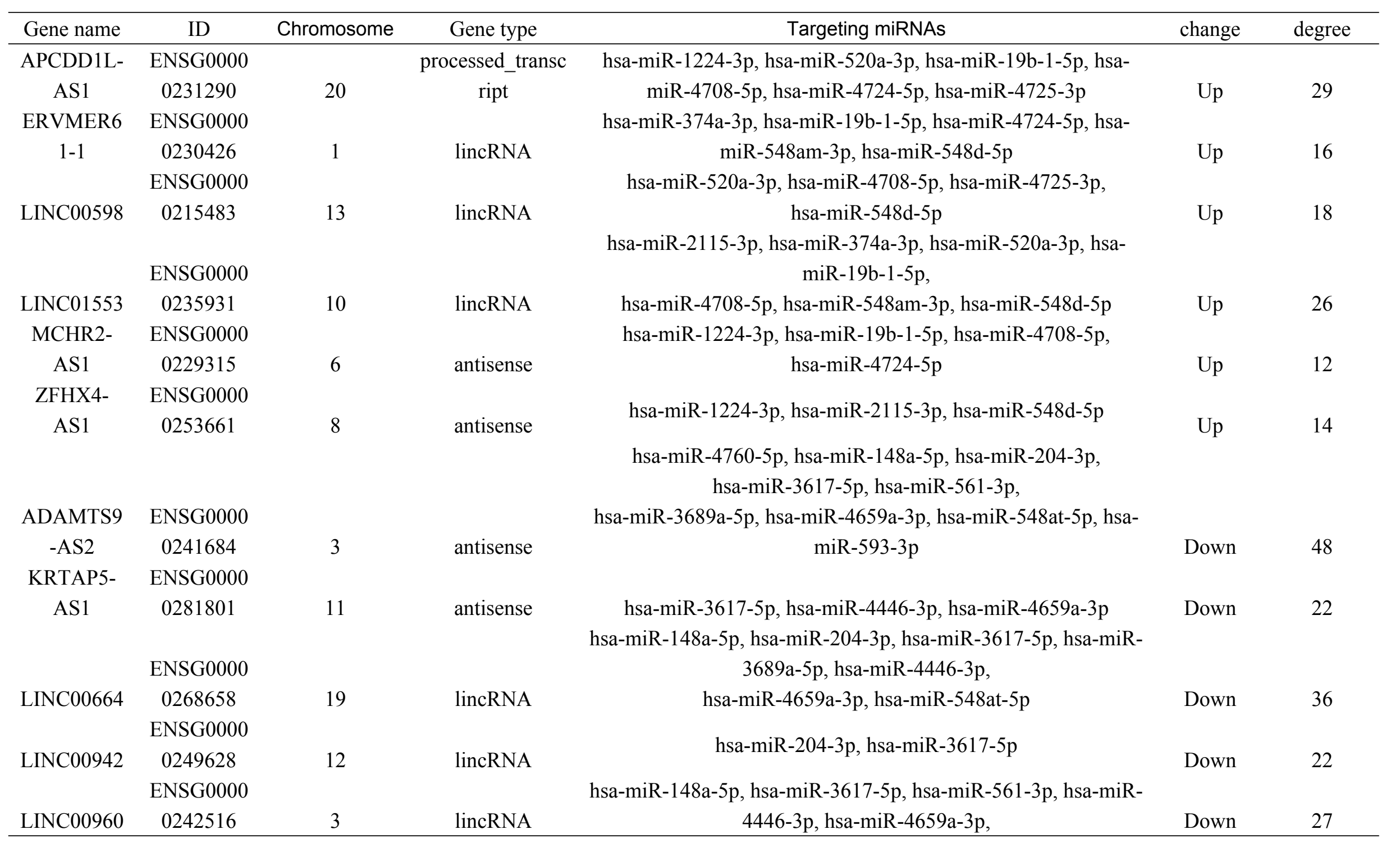


hsa-miR-548at-5p, hsa-miR-593-3p

hsa-miR-148a-5p, hsa-miR-204-3p, hsa-miR-3617-5p, hsa-miR-

0242808

sense_overlappi

561-3p, hsa-miR-3689a-5p,

hsa-miR-4659a-3p, hsa-miR-548at-5p, hsa-miR-593-3p

Down

56 
Table 5(on next page)

The top 5 predictive power of hub miRNA/mRNAs associated with response of esophageal squamous cell carcinomas to neoadjuvant chemoradiotherapy

The area under curve, sensitivity and specificity of significantly altered miRNA/mRNAs were summarized 
1

\begin{tabular}{lrrr}
\hline \multirow{2}{*}{ symbol } & \multirow{2}{*}{ auc } & specificit & sensitivity \\
\hline hsa-miR-4460 & 0.909 & 0.765 & 1 \\
hsa-miR-369-3p & 0.906 & 0.765 & 0.909 \\
hsa-miR-581 & 0.898 & 1 & 0.818 \\
hsa-miR-4524a-5p & 0.898 & 0.941 & 0.818 \\
hsa-miR-548ap-3p & 0.893 & 0.765 & 1 \\
QSOX2 & 0.92 & 1 & 0.727 \\
AZIN2 & 0.914 & 0.765 & 0.909 \\
RELL2 & 0.914 & 0.941 & 0.909 \\
EPHB2 & 0.914 & 0.765 & 0.909 \\
MEAF6 & 0.914 & 0.882 & 0.818 \\
\hline
\end{tabular}

2 


\section{Figure 1 (on next page)}

Heatmap representing the expression levels of genes between responder and nonresponder samples

A, heatmap of the top 100 mRNA of GSE45670. B, heatmap of the top 100 miRNA in GSE59974 
A

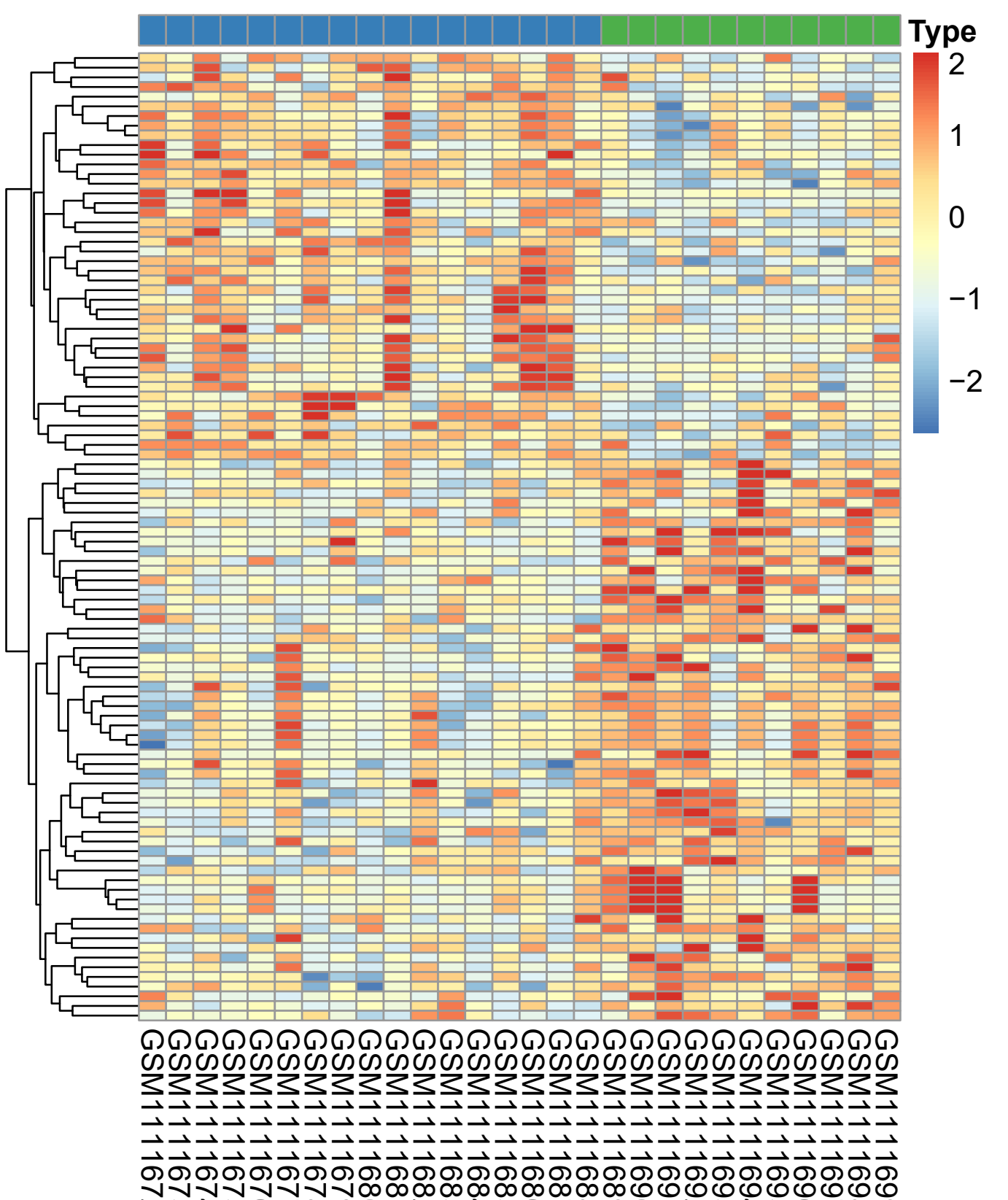

B

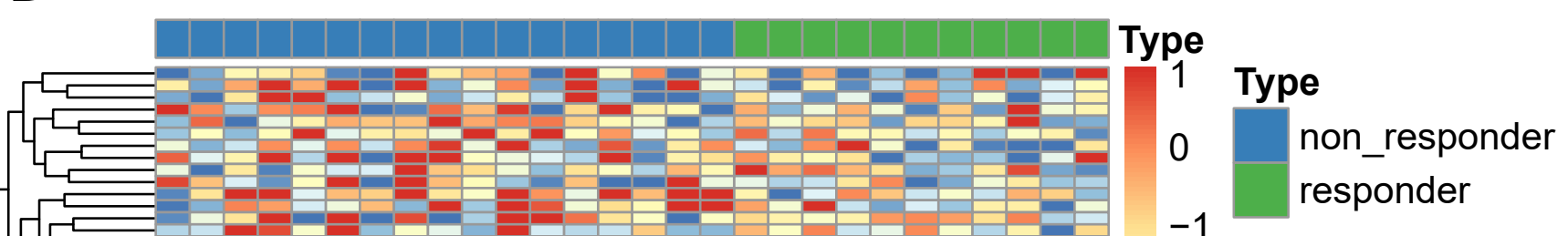
N $\omega$ A 
Figure 2 (on next page)

Gene ontology analysis of differentially expressed genes associated with response of esophageal squamous cell carcinomas to neoadjuvant chemoradiotherapy

A total of 145 mRNAs were chosen for $\mathrm{GO}$ analysis for biological process, cellular component and molecular function. The size of circle represented the gene counts in go terms, the colour represented the $\mathrm{p}$-value of go terms. A, biological process. B, cellular component. C, molecular function 

negative regulation of hydrolase activity

telencephalon development multicellular organism metabolic process cornification

multicellular organismal macromolecule metabolic process regulation of odontogenesis of dentin-containing tooth skeletal muscle adaptation regulation of odontogenesis bile acid and bile salt transport

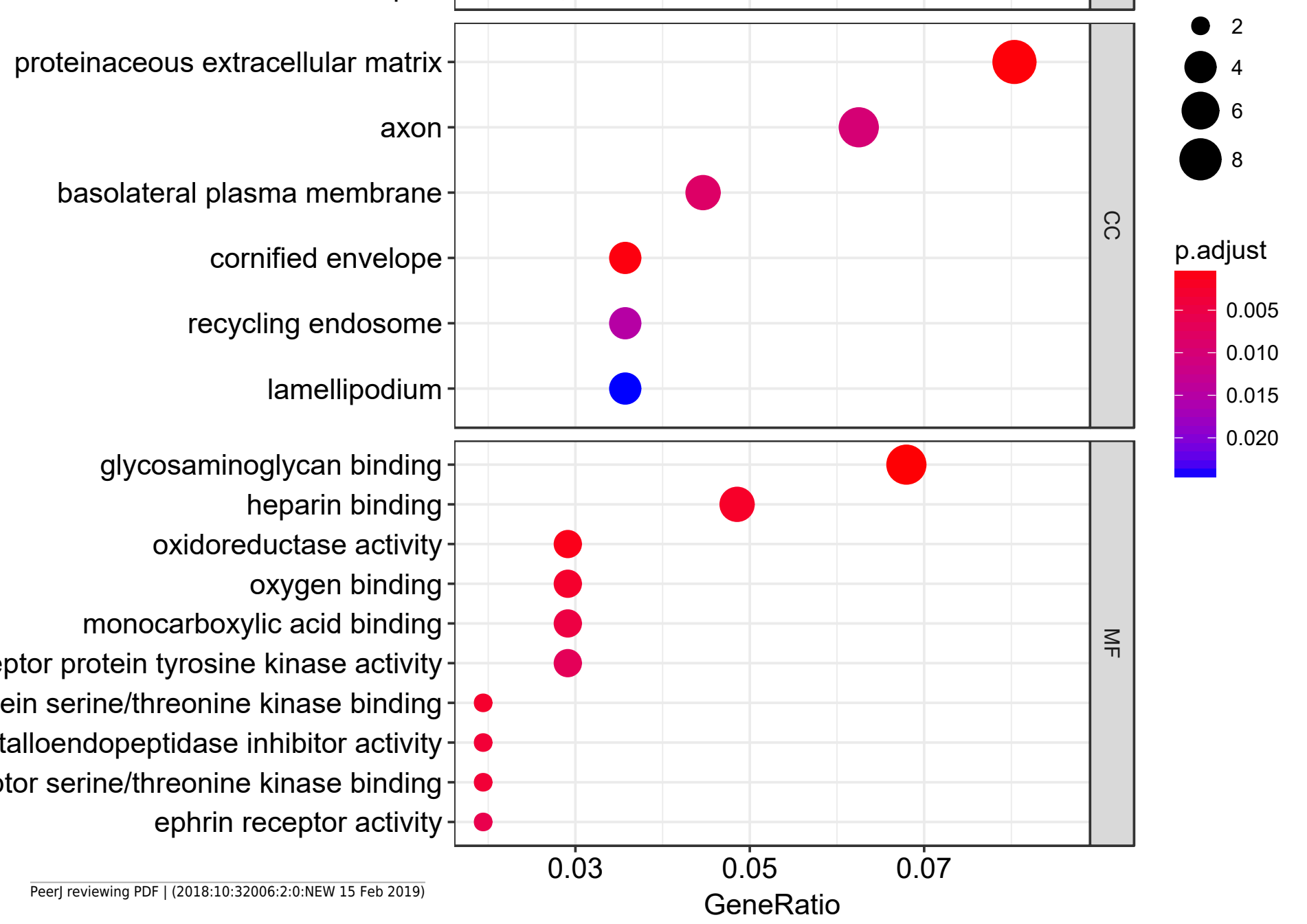

transmembrane receptor protein tyrosine kinase activity transmembrane receptor protein serine/threonine kinase binding metalloendopeptidase inhibitor activity receptor serine/threonine kinase binding ephrin receptor activity 


\section{Figure 3 (on next page)}

KEGG pathway analysis of differentially expressed genes associated with response of esophageal squamous cell carcinomas to neoadjuvant chemoradiotherapy.

A total of 145 mRNAs were chosen for kegg analysis. the color represented the $p$ value of kegg terms 
Metabolism of xenobiotics by cytochrome P450

Parathyroid hormone synthesis, secretion and action -

Axon guidance

\begin{abstract}
Neurotrophin signaling pathway
Cell adhesion molecules (CAMs) -
\end{abstract}

Celladhesion molecules (CAMs)

Wnt signaling pathway -
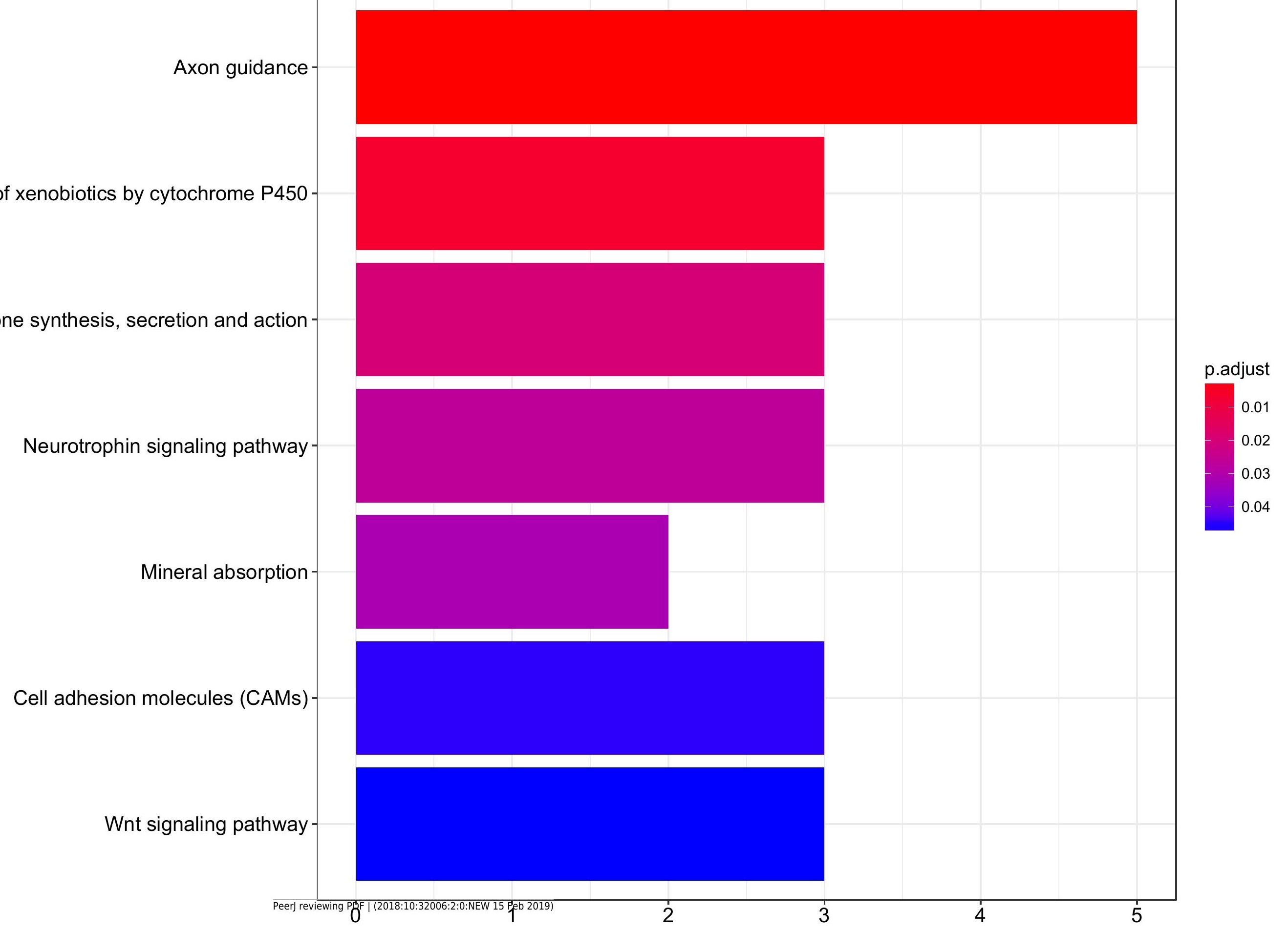


\section{Figure 4 (on next page)}

Protein-protein interaction between differentially expressed genes.

A total of 49 proteins interacted with each other. The size of the circle in the network represents the number of connections, red represents an up-regulated gene, blue represents a down-regulated expression gene, and purple frame represents a transcription factor. 


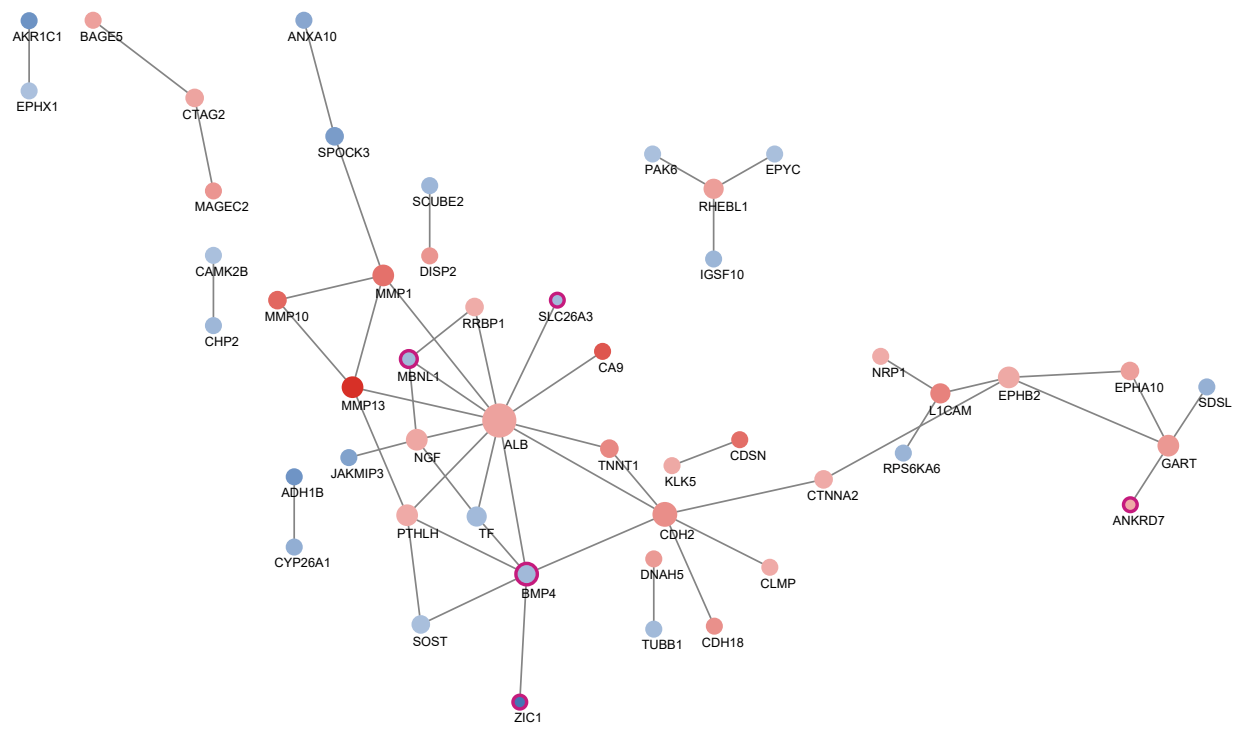


Figure $\mathbf{5}$ (on next page)

MiRNA regulation network associated with response of esophageal squamous cell carcinomas to neoadjuvant chemoradiotherapy

The size of the circle in the network represents the number of connections. pink represents high expression, blue represents low expression. The fill color represents mRNA. Outer frame color represents miRNA. A, 13 genes were regulated by two highly expressed hub miRNAs. B, 31 genes were regulated by the 5 low expressed hub miRNAs. 

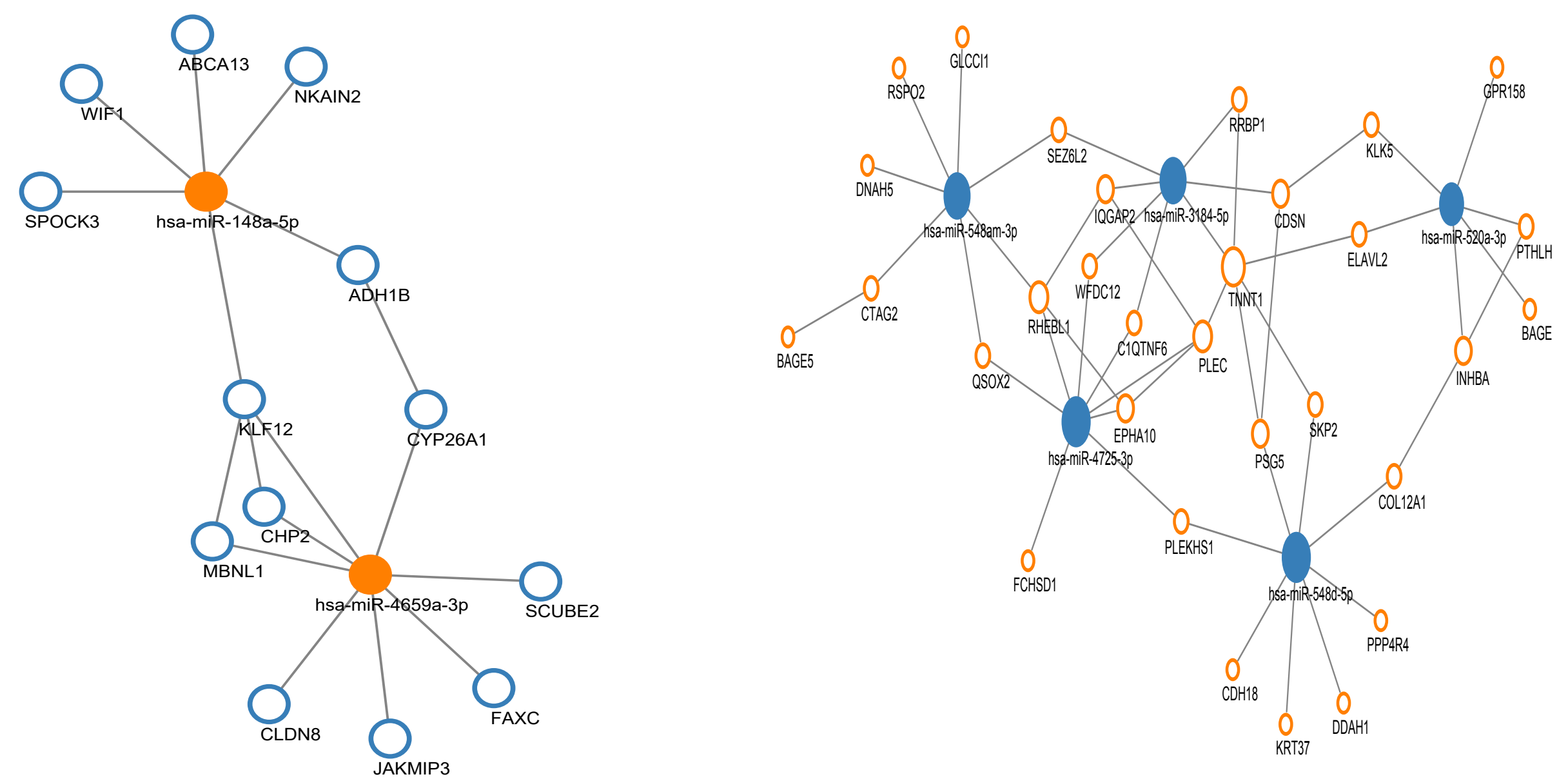
Figure $\mathbf{6}$ (on next page)

Competitive endogenous RNA (ceRNA) regulation network associated with response of esophageal squamous cell carcinomas to neoadjuvant chemoradiotherapy.

The size of the circle in the network represents the number of connections. pink represents high expression, blue represents low expression. Diamonds represent miRNAs. The fill color of the circle represents IncRNA. The outer frame color represents mRNA. A, 6 highly expressed IncRNAs regulate 10 miRNAs and 44 genes. B, 6 highly expressed IncRNAs regulate 10 miRNAs and 27 genes. 

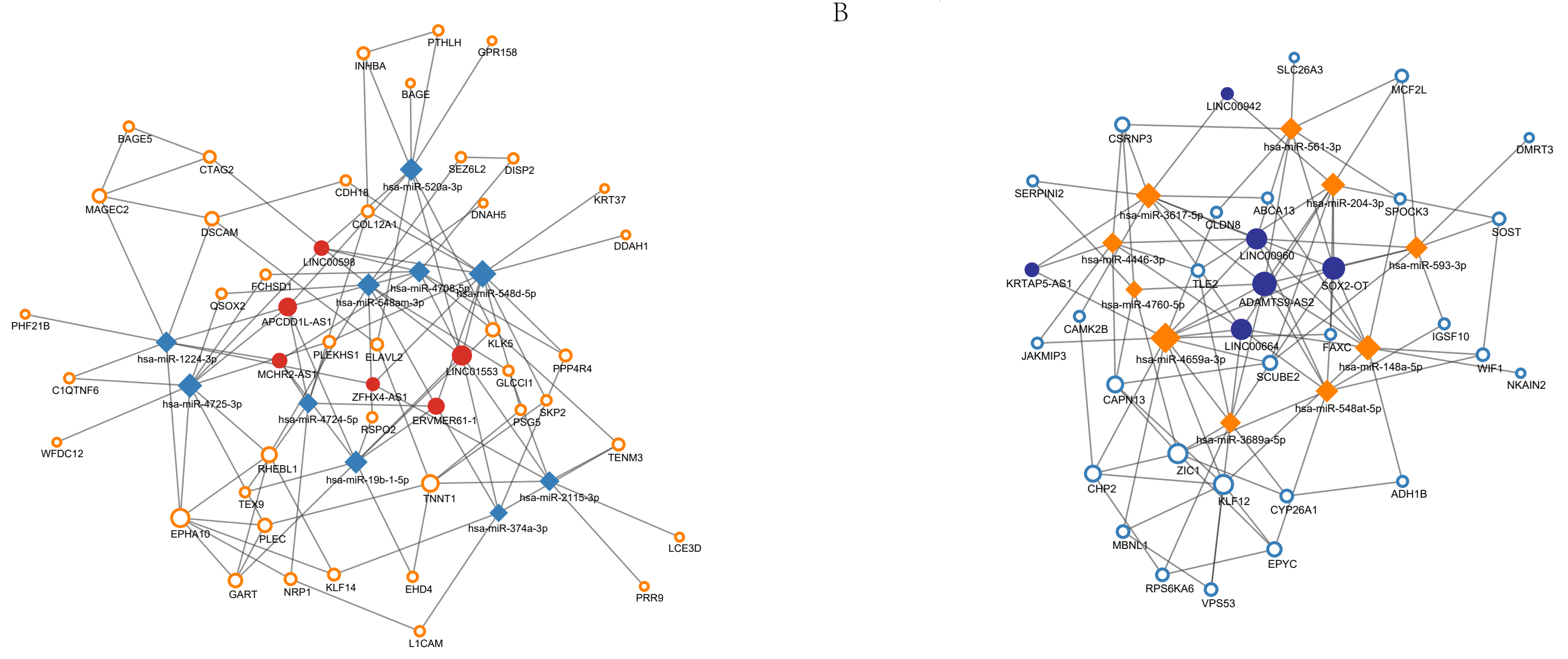